\title{
Bell's palsy following a single dose of mRNA SARS-CoV-2 vaccine: a case report
}

\author{
C. Martin-Villares ${ }^{1}$ (D) A. Vazquez-Feito ${ }^{2} \cdot$ M. J. Gonzalez-Gimeno ${ }^{3} \cdot$ B. de la Nogal-Fernandez ${ }^{4}$
}

Received: 12 April 2021 / Revised: 13 May 2021 / Accepted: 18 May 2021 / Published online: 25 May 2021

c) Springer-Verlag GmbH Germany, part of Springer Nature 2021

\section{Dear Editor,}

During pandemic influenza A (H1N1) vaccination campaign in 2009, relative risk for Bell's palsy (BP) following vaccine increased significantly [1]. Combining data from both SARS-CoV-2 Pfizer-BioNTech and Moderna vaccine trials, Ozonoff et al. [2] suggest an imbalance in the incidence of BP following vaccination (seven cases) compared with the placebo group (one case). The US Food and Drug Administration (FDA) pointed out that a causal relationship between the COVID-19 vaccines and BP cannot be definitively excluded and consequently, recommended strict pharmacovigilance for BP after COVID-19 vaccination in large populations [3].

In February 2021, we had the opportunity to treat a 34-year-old woman who developed a right facial palsy following Moderna COVID-19 vaccine. She developed a right Bell's palsy in 2012 during pregnancy (5th month) but not during her second pregnancy (2017). On February 12, our patient received her first dose of the Moderna vaccine. On February 14 (2nd day), she developed right facial pain with

C. Martin-Villares

crismvillares@gmail.com

A. Vazquez-Feito

lacianiego6522@gamil.com

M. J. Gonzalez-Gimeno

mjgonzalezgimeno@gmail.com

B. de la Nogal-Fernandez

mnogal@saludcastillayleon.es

1 ENT Department, Hospital El Bierzo, University of LeonCampus of Ponferrada, Leon, Spain

2 Clinical Neurophysiology Department, Hospital El Bierzo, University of Leon-Campus of Ponferrada, Leon, Spain

3 ENT Department, Hospital Infanta Sofia, Universidad Europea, Madrid, Spain

4 Hospital Pharmacist, Hospital El Bierzo, University of Leon-Campus of Ponferrada, Leon, Spain a House-Brackmann grade III facial palsy. The SARS-CoV-2 PCR test was negative in nasal swab, and IgG Varicella Zoster Virus (VZV) Antibodies were positive in serology. Only lymphocytosis was relevant in the blood count. Deflazacort $30 \mathrm{mg} /$ day, eye support and facial rehabilitation were prescribed. No relevant findings were identified in MRI study. Nerve conduction studies (Fig. 1) showed an axonal facial neuropathy with a drop of CMAP $82 \%$ for mandibular branch, 66\% for nasalis branch and $80 \%$ for zygomatic branch compared to the contralateral branch. Facial palsy began to improve on February 22 (10th day) and finally on March 4, full recovery was achieved. The second dose of the vaccine was not administrated to our patient. The patient had developed a previous facial palsy during pregnancy in 2012 and in February 2021, the only information available on facial paralysis after the first dose of mRNA vaccine was one patient who developed a FP during Pfizer-BioNTech vaccine trial, and the patient did not receive a second dose [4]. Four weeks after the first dose of the vaccine, our patient was tested positive for IgG SARS-CoV-2 antibodies. In postauthorization phase, Colella et al. [5] have reported the first case of BP palsy after Pfizer-BioNTech vaccine on fifth day, but to our knowledge, there are no reports of FP after the first dose of Morderna vaccine.

In the Moderna trial report [3], the researchers considered the temporal association and the biological plausibility between the vaccine and the BP, but they suggested that causality assessment might be confounded by predisposing factors. In our young and healthy patient, a previous facial palsy during pregnancy and the presence of VZV-IgG antibodies could be associated with an increased predisposition to facial nerve palsy, but evidence of a temporal association between the vaccine administration and the facial nerve palsy is clear: Bell's palsy appeared 2 days after the administration of the mRNA COVID-19 vaccine. 


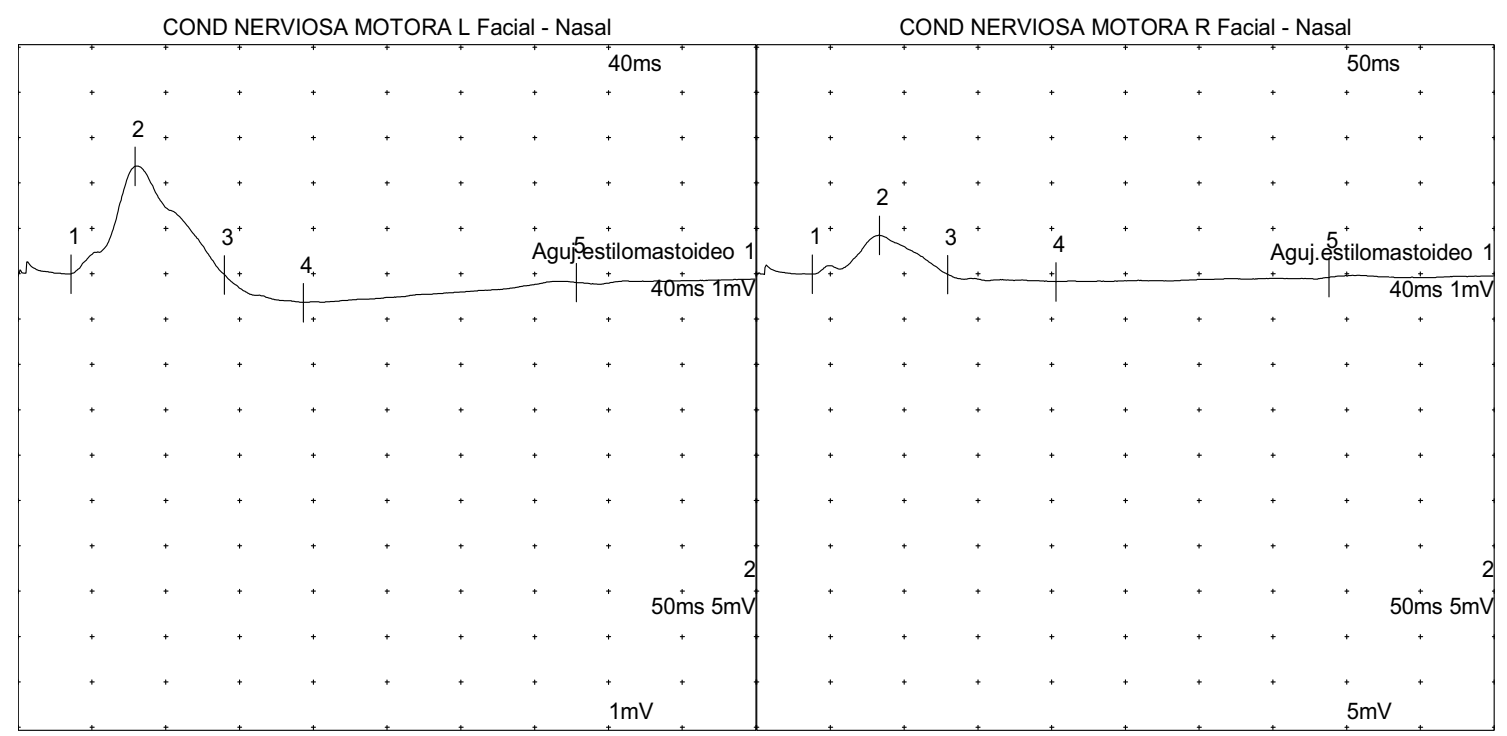

Fig. 1 Compound muscle action potential of facial nerve spared vs affected in nerve conduction studies with conventional technique [6]

\section{Declarations}

Conflicts of interest None of the authors declare any conflict of interests.

Ethical approval The patient gave explicit informed consent to report her clinical case, and the adverse effect was communicated to the national health authorities.

\section{References}

1. Bardage C, Persson I, Ortqvist A, Bergman U, Ludvigsson JF, Granath F (2011) Neurological and autoimmune disorders after vaccination against pandemic influenza A (H1N1) with a monovalent adjuvanted vaccine: population based cohort study in Stockholm, Sweden. BMJ 12(343):d5956. https://doi.org/10. 1136/bmj.d5956 (PMID: 21994316; PMCID: PMC3192001)

2. Ozonoff A, Nanishi E, Levy O (2021) Bell's palsy and SARSCoV-2 vaccines. Lancet Infect Dis. https://doi.org/10.1016/S14733099(21)00076-1 (PMID: 33639103; PMCID: PMC7906673)

3. US Food and Drug Administration (2020) Moderna COVID19 vaccine emergency use authorization review memorandum. https://www.fda.gov/media/144673/download

4. US Food and Drug Administration (2020) Pfizer-BioNTech COVID-19 Vaccine Emergency Use Authorization Review Memorandum. https://www.fda.gov/media/144416/download

5. Colella G, Orlandi M, Cirillo N (2021) Bell's palsy following COVID-19 vaccination. J Neurol. https://doi.org/10.1007/ s00415-021-10462-4

6. Dumitru D, Walsh NE, Porter LD (1988) Electrophysiologic evaluation of the facial nerve in Bell's palsy. A review. Am J Phys Med Rehabil 67(4):137-144 